\title{
Incidentally Discovered Cor Triatriatum Sinistrum Anomaly During Heart Transplant
}

\author{
Magdy El-Sayed Ahmed ${ }^{1}$, Ian Makey ${ }^{1}$, Mathew Thomas ${ }^{1}$, Samuel Jacob², Osama Haddad ${ }^{1}$, \\ Basar Sareyyupoglu ${ }^{1}$, Kevin Landolfo ${ }^{1}$, Parag Patel $^{2}$, and Si Pham ${ }^{1}$ \\ ${ }^{1}$ Mayo Clinic Florida \\ ${ }^{2}$ Mayo Clinic
}

July 16, 2020

\section{Incidentally Discovered Cor Triatriatum Sinistrum Anomaly During Heart Transplant Cor Triatriatum Discovered During Heart Transplant}

Magdy M. El-Sayed Ahmed, MD, MS ${ }^{1,3}$;Ian A. Makey, MD ${ }^{1}$; Mathew Thomas, MD ${ }^{1}$; Samuel Jacob, MD ${ }^{1}$; Osama Haddad, MD ${ }^{1}$; Basar Sareyyupoglu, M.D ${ }^{1}$; Kevin P. Landolfo, MD, MS ${ }^{1}$; Parag C Patel, MD ${ }^{2}$; Si M. Pham, MD, FACS ${ }^{1}$

Department of Cardiothoracic Surgery ${ }^{1}$, Transplantation ${ }^{2}$, Mayo Clinic, Jacksonville, FL Department of Surgery ${ }^{3}$, Zagazig University Faculty of Medicine, Zagazig, Egypt

\section{Corresponding author}

Magdy M. El-Sayed Ahmed

Mail address: 4500 San Pablo Rd S, Jacksonville, FL 32224

Email:ahmed.magdy@mayo.edu

Phone: 904-953-2190

Fax: 904-953-73

Cor triatriatum is abnormal septation within the atrium. It exits in two different varieties; cor triatriatum sinister (CTS) in the left atrium and cor triatriatum dexter (CTD) in the right atrium. Cor triatriatum sinister is an uncommon congenital cardiac anomaly with an incidence of about $0.1-0.4 \%$ of patients with congenital cardiac disease. CTS usually associated with other congenital cardia anomalies such as ostium secondum, patent foramen oval, atrial septal defect, left superior vena cava, anomalous pulmonary venous return, and mitral valve regurgitation. CTD is commonly associated with right ventricular hypoplasia, pulmonary valve atresia, pulmonary valve stenosis, and Ebstein anomaly. Symptoms typically mimic mitral and tricuspid in CTS and CTD respectively. Surgical resection is the standard treatment, however, balloon dilatation may be considered in certain circumstances as heart failure, pregnancy or as a bridge to definitive management.

We report an incidentally discovered CTS during heart transplant in a 51-year-old male patient with heart failure due to ischemic cardiomyopathy. He had been experiencing intermittent angina. His past medical and surgical history were remarkable for coronary stenting and coronary artery bypass grafting. His echocardiography revealed a thin band-like structure in the left atrium (Fig. 1A). A suitable donor was available and the patient successfully underwent orthotopic heart transplant. During explanation of the native heart, CTS was 
discovered as a semicircular membrane within the left atrium (Fig. 1B). CTS was completely excised and careful inspection of the pulmonary veins and both superior and inferior vena cavae showed no anomalies. His postoperative course was uneventful and he was discharged from the hospital on postoperative day 13.

Although CTS is a rare congenital cardiac anomaly, adult cardiac surgeons should be aware of the potential associated other anomalies specially left superior vena cava and anomalous pulmonary venous return during heart transplant procedure.

\section{Conflict of interest disclosure}

The authors have no conflict of interest and this study was not financially supported

\section{Figure Legends}

Fig. 1: (A) TTE shows a band within the left atrium (arrow) and mitral valve (star), and (B) Photo shows the cor triatriatum (forceps), the aorta (star), the main pulmonary artery (arrow), and the superior vena cava cannula (arrow head).

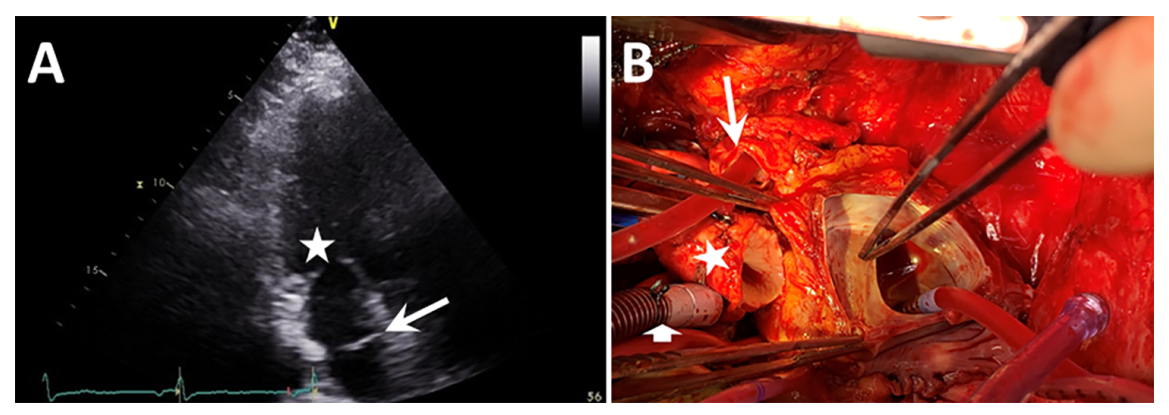

